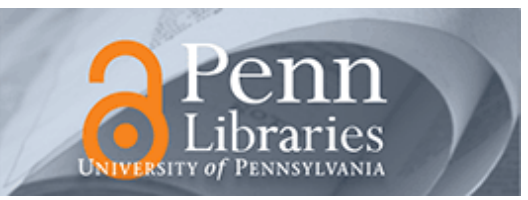

University of Pennsylvania

ScholarlyCommons

\title{
Free-Standing Leaping Experiments with a Power-Autonomous, Elastic-Spined Quadruped
}

\author{
Jason L. Pusey \\ Army Research Laboratory \\ Jeffrey M. Duperret \\ University of Pennsylvania, jdup@seas.upenn.edu
}

\section{G. Clark Haynes \\ National Robotic Center}

Rof

'I-iversity of Pennsylvania

Part of the Electrical and Computer Engineering Commons, and the Systems Engineering Commons

Daniel E. Koditschek

University of Pennsylvania, kod@seas.upenn.edu

Recommended Citation

Jason L. Pusey, Jeffrey M. Duperret, G. Clark Haynes, Ryan Knopf, and Daniel E. Koditschek, "Free-

Standing Leaping Experiments with a Power-Autonomous, Elastic-Spined Quadruped", . April 2013.

\section{BibTeX entry}

@article\{canid_spie_2013, author $=\{$ Pusey, Jason L. and Duperret, Jeffrey M. and Haynes, G. Clark and Knopf, Ryan and Koditschek, Daniel E. $\}$, title $=\{$ Free-Standing Leaping Experiments with a Power-Autonomous, Elastic-Spined

Quadruped $\}$, pages $=\{87410 \mathrm{~W}-87410 \mathrm{~W}-15\}$, year $=\{2013\}, \mathrm{doi}=\{10.1117 / 12.2016073\}\}$

This work is supported by the National Science Foundation Graduate Research

Fellowship under Grant Number DGE-0822, and by the Army Research

Laboratory under Cooperative Agreement Number W911NF-10-2-0016.

Copyright 2013 Society of Photo-Optical Instrumentation Engineers. Postprint version.

This paper was (will be) published in Proceedings of the SPIE Defense, Security, and Sensing Conference,

Unmanned Systems Technology XV (8741), and is made available as an electronic reprint with permission of SPIE.

One print or electronic copy may be made for personal use only. Systematic or multiple reproduction, distribution to multiple locations via electronic or other means, duplication of any material in this paper for a fee or for commercial purposes, or modification of the content of the paper are prohibited.

This paper is posted at ScholarlyCommons. https://repository.upenn.edu/ese_papers/655

For more information, please contact repository@pobox.upenn.edu. 


\title{
Free-Standing Leaping Experiments with a Power-Autonomous, Elastic-Spined Quadruped
}

\author{
Abstract \\ We document initial experiments with Canid, a freestanding, power-autonomous quadrupedal robot \\ equipped with a parallel actuated elastic spine. Research into robotic bounding and galloping platforms \\ holds scientific and engineering interest because it can both probe biological hypotheses regarding \\ bounding and galloping mammals and also provide the engineering community with a new class of agile, \\ efficient and rapidly-locomoting legged robots. We detail the design features of Canid that promote our \\ goals of agile operation in a relatively cheap, conventionally prototyped, commercial off-the-shelf \\ actuated platform. We introduce new measurement methodology aimed at capturing our robot's "body \\ energy" during real time operation as a means of quantifying its potential for agile behavior. Finally, we \\ present joint motor, inertial and motion capture data taken from Canid's initial leaps into highly energetic \\ regimes exhibiting large accelerations that illustrate the use of this measure and suggest its future \\ potential as a platform for developing efficient, stable, hence useful bounding gaits. \\ For more information: Kod* Lab \\ Disciplines \\ Electrical and Computer Engineering | Engineering | Systems Engineering

\section{Comments} \\ BibTeX entry \\ @article\{canid_spie_2013, author = \{Pusey, Jason L. and Duperret, Jeffrey M. and Haynes, G. Clark and \\ Knopf, Ryan and Koditschek, Daniel E. $\}$, title $=\{$ Free-Standing Leaping Experiments with a Power- \\ Autonomous, Elastic-Spined Quadruped $\}$, pages $=\{87410 \mathrm{~W}-87410 \mathrm{~W}-15\}$, year $=\{2013\}$, doi $=\{10.1117 /$ \\ $12.2016073\}$ \} \\ This work is supported by the National Science Foundation Graduate Research \\ Fellowship under Grant Number DGE-0822, and by the Army Research \\ Laboratory under Cooperative Agreement Number W911NF-10-2-0016. \\ Copyright 2013 Society of Photo-Optical Instrumentation Engineers. Postprint version. \\ This paper was (will be) published in Proceedings of the SPIE Defense, Security, and Sensing Conference, \\ Unmanned Systems Technology XV (8741), and is made available as an electronic reprint with permission \\ of SPIE. \\ One print or electronic copy may be made for personal use only. Systematic or multiple reproduction, \\ distribution to multiple locations via electronic or other means, duplication of any material in this paper \\ for a fee or for commercial purposes, or modification of the content of the paper are prohibited.
}




\title{
Free-Standing Leaping Experiments with a Power-Autonomous, Elastic-Spined Quadruped \\ Jason L. Pusey ${ }^{\text {a }}$, Jeffrey M. Duperret ${ }^{b}$, G. Clark Haynes ${ }^{c}$, Ryan Knopf ${ }^{b}$, and Daniel E. Koditschek ${ }^{b}$ \\ ${ }^{a}$ U.S. Army Research Laboratory, Aberdeen Proving Ground, MD, \\ ${ }^{\mathrm{b}}$ University of Pennsylvania, Philadelphia, PA, \\ ${ }^{\mathrm{c}}$ National Robotics Engineering Center, Carnegie Mellon University, Pittsburgh, PA
}

\begin{abstract}
We document initial experiments with Canid, a freestanding, power-autonomous quadrupedal robot equipped with a parallel actuated elastic spine. Research into robotic bounding and galloping platforms holds scientific and engineering interest because it can both probe biological hypotheses regarding bounding and galloping mammals and also provide the engineering community with a new class of agile, efficient and rapidly-locomoting legged robots. We detail the design features of Canid that promote our goals of agile operation in a relatively cheap, conventionally prototyped, commercial off-the-shelf actuated platform. We introduce new measurement methodology aimed at capturing our robot's "body energy" during real time operation as a means of quantifying its potential for agile behavior. Finally, we present joint motor, inertial and motion capture data taken from Canid's initial leaps into highly energetic regimes exhibiting large accelerations that illustrate the use of this measure and suggest its future potential as a platform for developing efficient, stable, hence useful bounding gaits.
\end{abstract}

Keywords: Legged Robot, Modular robot, Bounding quadruped, Flexible Spine, Robot design

\section{INTRODUCTION}

This paper discusses design improvements in the Canid platform [20] that have resulted in free-standing, power autonomous, repeatable open loop leaping behavior approaching the realm of a sustained bounding gait. We focus on those few design innovations - a parallel elastic actuated flexible spine; and a four-bar leg mechanism that distinguish the platform from its otherwise identically constructed relative, the rigid-backed XRL platform [20], the latest update of the decade old RHex [43] design. We present as well some of the new measurement methodology we have developed to facilitate the characterization of "body energy" in both of these robots since we hypothesize that this quantity may provide a key signature of agility. Finally, we document the present state of Canid's leaping behavior, illustrating the use of this measure, summarizing the behavioral advances it portends, and identifying the key technical obstacles whose resolution we believe will yield stable, sustained, robust, efficient bounding. We give further details and analysis of Canid's leaping behavior in [14].

The term "body energy" denotes the sum of mechanical energies attributable to point mass approximations of the major rigid bodies that comprise a mechanical system along with the potential energies in the elastic elements that couple them (see the discussion around Figure 7 in Section 3.2.2 for the working definition we apply here). This represents an empirically accessible measure of a machine's readiness to perform rapid work on its environment or its mass center within the environment. Whereas fuel energy requires conversion through an actuator at a rate limited by its inevitably constrained power capacity [25], kinetic energy can, in stance, be immediately redirected by leg-steered ground reaction forces and potential energy can be released at rates limited only by the body's dynamical state. Of course, while a highly energetic regime may be necessary for agility in conventionally actuated machines, it is obviously not sufficient: purposeless flailing of limbs and body will achieve high body energy but very little useful work, or, perhaps, work only at very high cost. We find (and document in a paper now under review [15]), that Canid's design promotes such highly energetic operation without undue losses.

In the longer term, we believe that Canid is interesting not because it is so different from XRL but because it is so similar. Canid's novel spine and RiSE-inspired [46] legs represent the only real differences: most importantly, the two machines have the same number of the same actuators and drive electronics so their power budget is identical. Yet it is already clear (as we document in [15]) that Canid operates far more energetically with little adverse impact on efficiency relative to XRL. The question remains whether even this pairing (more energetic and efficient) is really 
sufficient for greater agility. XRL is a generalist ${ }^{1}$ and like all the machines in the RHex lineage [43], it is capable of multiple gaits [35], [36], [37], [8], rapid transitions between them [26] and even a great diversity of highly energetic, useful leaps [27]. In contrast, Canid is a specialist: we are skeptical that it will efficiently sustain other dynamical quadrupedal gaits (e.g., trotting or pacing) much less ever operate in the quasi-static regime because there is no control authority over torsion (coronal plane bending) in the spine. By adding degrees of freedom and a new elastic coupling between them, we have exposed more dynamics in Canid, hence the ready prospect of higher operating energies. But, even if they can be tamed for efficiency in steady state, it remains to be seen if their control can be rendered as capably in transitional behaviors as that of RHex. In this regard, the promotion of careful systems level comparisons between component wise comparable designs, we believe that the "Laboratory on Legs" concept [20] may in the long run prove as interesting as Canid or XRL themselves.

\subsection{Background: Flexible Spine Quadrupedal Bounding}

It is generally accepted that many quadrupedal mammals obtain their highest locomotion speeds through a bounding or galloping gait [24]. The Cheetah, considered the fastest running mammal on earth, and the greyhound, both with similar lean musculoskeletal structures, make extensive use their spine during high speed running [5], [23]. The flexion excitation of the spine systematically coordinated with the swing of the legs, together provide kinematic advantages in increased stride length and the combined forces of the spine and leg increase swing acceleration both supporting faster speeds when compared to the use of the legs alone [23]. The relationship between the high powered legs and strong flexible spine and their collective ability to store and release energy to achieve high speeds motivate Canid's design concept.

Initial bounding robot research focused on leg design and how to achieve stable behaviors using legs on a rigid body. Scout II, a quadruped with a rigid body and actuated pogo sticks (hip torque, passive prismatic springs) for legs, achieved one of the earliest free-standing, power-autonomous instances of stable bounding [40], [45]. BigDog, a much larger platform created by Boston Dynamics, is also able to achieve stable bounding behaviors with a fixed spine and multiple degree of freedom legs, which are hydraulically actuated [6]. Probably the very first flexible-spined bounding robot was the boom-supported "Planar Quadruped" developed in Raibert's leg lab [31], which achieved highly energetic gaits (speeds of up to $\sim 70 \mathrm{~cm} / \mathrm{s}$ in a $\sim 90 \mathrm{~cm}$ long body) forced by off board (hydraulic) powered actuators. Subsequent research has focused on the role of bioinspired gait in flexibly spined legged platforms. For example, ELIRO-II incorporates a two degree of freedom spine to promote study of the salamander walking gait control [29], [39]. Salamander locomotion has also inspired the development of central pattern generators (CPGs) and reflex controllers for multi-locomotors gaits such as swimming, crawling, and walking [1], [7], [10], as well as purely leg driven flexible spined gaits [47]. Other more mammalian bioinspiration for quadrupedal robots with articulated spinal mechanical joints include GEO-II [32], [33], and BISAM [4], used to develop and explore CPG controllers. In contrast to our focus on Canid's energetics, none of these rigid link spinal joint mechanisms is designed for operation in regimes of high kinetic energy: they do not incorporate mechanisms for recycling energy absorbed into the articulated trunk from locomotive contact with the environment and appear limited to operation in a quasi-static regime.

In more recent years, simulation studies have begun to suggest that a flexible spine confers several dynamical advantages over rigid body bounding [9], [11], [12], [13], [21], [41]. In particular, results from, [12], [9], suggest that for about the same specific resistance, a quadruped with an actuated spine might leap higher and farther than a fixed spine design. In turn, the past two years, have witnessed the introduction of a far more dynamical family of flexible spine robot quadrupeds. A fascinating study of a purely passive, gravity-driven, elastic-spined quadruped capable of long aerial phased downhill bounding suggests that bidirectional sagittal spine compliance may promote more stable and faster gaits than can a rigid or unidirectional (downward bending only) spine [28]. Because Canid was designed to explore just this transfer of energy between the legs and spinal element relative to their role in promoting efficiency and speed, we are happy that bidirectional operation is available, but we have not yet seen much evidence of its appearance in the preliminary leaping data we present here. Canid's powerful flexible spine is specifically motivated by evolutionary evidence that vertebrate legs first appeared as spring struts primarily actuated by strong, segmented,

\footnotetext{
${ }^{1}$ There is a long tradition in evolutionary ecology of comparing specialist and generalist strategies in animal locomotion [22] (and other behaviors) in order to better understand their relationship between form, function, and fitness. Similar consideration s hold key importance in robotics and we look forward to the possibility that the enhanced quantitative traditions of engineering promoted by relative ease of measurement in physical models of locomotion (e.g. Canid, XRL) may stimulate a useful interchange of ideas about such relationships with biologists.
} 
undulatory trunks responsible for propulsion as animals emerged from the sea [17]. This same hypothesis is explored with greater purity of focus in the recent Kitty design [30], [49], which features a richly parameterized family of cabledriven compliant spine assemblies permitting both sagittal and coronal plane bending and with no other actuation at all. However, this machine's operating dynamics appears to be largely concentrated in the spine itself (apparently the central focus of its authors' interesting information theoretic classification of gait patterns) with locomotion of its $\sim 30 \mathrm{~cm}$ long body reported at roughly 0.2 body lengths per second $(\sim 6 \mathrm{~cm} / \mathrm{s})$ bounding and 0.1 body lengths per second $(\sim 3 \mathrm{~cm} / \mathrm{s})$ trotting. In contrast, Canid is a specialized, complete body dynamics obligate ${ }^{2}$ : energy necessarily courses through its entire body at every step and its locomotory style is an order of magnitude faster with locomotion of its $\sim 80 \mathrm{~cm}$ long body reported here at roughly 2 body lengths per second (see Table 2).

The most highly energetic of flexible spined quadrupeds in contemporary robotics may be the hydraulically driven Cheetah design by Boston Dynamics [6], which may have claimed on the record for land running robot speed (although it seems still to be supported by a sagittal plane boom). At the forefront of contemporary bioinspired mechanism design, we await with keen interest behavioral reports on the novel MIT Cheetah robot whose legs are engineered with revolutionary structural rigidity, and unusually efficient, power-dense actuation designed to support and drive the robot in high energy and ultra-high force regimes [1], [16], [18], [44], [34], [48]. Although data from these machines permitting such comparison is still unavailable, we suspect that neither design emphasizes the role of the spine in high powered thrusting and energy harvesting throughout a stride as the data we present below show to be true of Canid's operation. In this regard, Canid seems to be unique in its ability to capture spine energetics to aid locomotion, and seems presently to hold the claim of the only free standing highly energetic flexibly spined bounding robot.

\subsection{Organization and Contributions}

Section 2, details the Canid robot with a report of evolutionary design upgrades and enhancements developed through iterative prototyping since its inception over two years ago [20]. Section 3 describes our laboratory methodology for measuring Canid's body energy, and then uses that methodology to present the first data on Canid's open loop bounding. Finally, Section 4 concludes with a brief summary and discussion of future directions.

\section{CANID}

\subsection{The Canid Robot Design}

Canid is a quadrupedal freestanding robotic platform equipped with an actuated elastic spine mechanism, created in collaboration between the U.S. Army Research Laboratory (ARL) and the University of Pennsylvania under support from the Robotics Collaborative Technology Alliance (R-CTA). The spine assembly consists of a carbon fiber flat plate actuated by two sets of cables located above and below, in parallel. When the cables are pulled, the spine acts as a leaf spring, storing energy as a function of its potentially bidirectional flexion displacement. Each leg is actuated by an independent motor through a four-bar mechanism inspired by the RiSE design [46]. The four-bar mechanism allows the robot to maintain roughly constant motor shaft speed and drive the legs' toe paths through a putatively efficient cycle without requiring additional actuators to shape it. The actual toe of the leg mechanism was adapted from the original RHex C-leg designs [43], [35], [36], [37]. The internal electronic components and software architecture of Canid are also built directly from the "Laboratory on Legs" infrastructure shared with the latest incarnation of the RHex design [20]. All these parts are held together by an aluminum space-frame chassis.

\subsubsection{An Actuated Elastic Spine}

Canid's single claim to a feature unique (to the best of our knowledge) within contemporary quadrupedal robotic design is its flexible carbon fiber leaf spring separating the front shoulders from the rear hips through a rigid connection at each end. Two sets of cables mounted parallel to the spine and offset from the neutral axis, both above and below, are connected to motors, extending and flexing the spine in a convex and concave manner, respectively. This design was inspired most directly by Zeglin's Bow Legged Hopper [50], which uses a cable actuated leaf spring leg compressing and releasing the spring to power the hopping motion. The goal is to enlist the robot's dynamic motion in harvesting and returning spring potential so as to promote its gait speed and efficiency.

\footnotetext{
${ }^{2}$ Once again in evolutionary ecology and developmental biology, the determination of obligate vs. facultative behavior as a function of morphology has a long tradition [42] that we in robotics can aspire both to learn from and contribute toward through more formal study of what locomotory strategies are either precluded or unavoidable in our designs.
} 
Repeated iterations on the initial design [20] have achieved a platform capable of successive open loop driven leaps sufficiently repeatable to permit the extended bounding we report on below. The most crucial of these redesign steps addressed an inadequately tuned original spine compliance that allowed undesirable twisting and overly large bending deflections. The redesigned leaf spring is thicker, wider, and shorter, maintaining necessary sagittal flexibility while significantly stiffening the torsional compliance.

Early experiments also revealed that inevitable disturbances accompanying intended operation (e.g., ground impact or rapid release at launch) would excite parasitic sagittal bending and even buckling in the leaf spring as actuated by its initial cable mechanism. Threading the cables through vertebral braces slipped onto the leaf spring (See the spine elements in Figure 1) significantly increased control over flexural loading and successfully enforced the desired spine curvature.

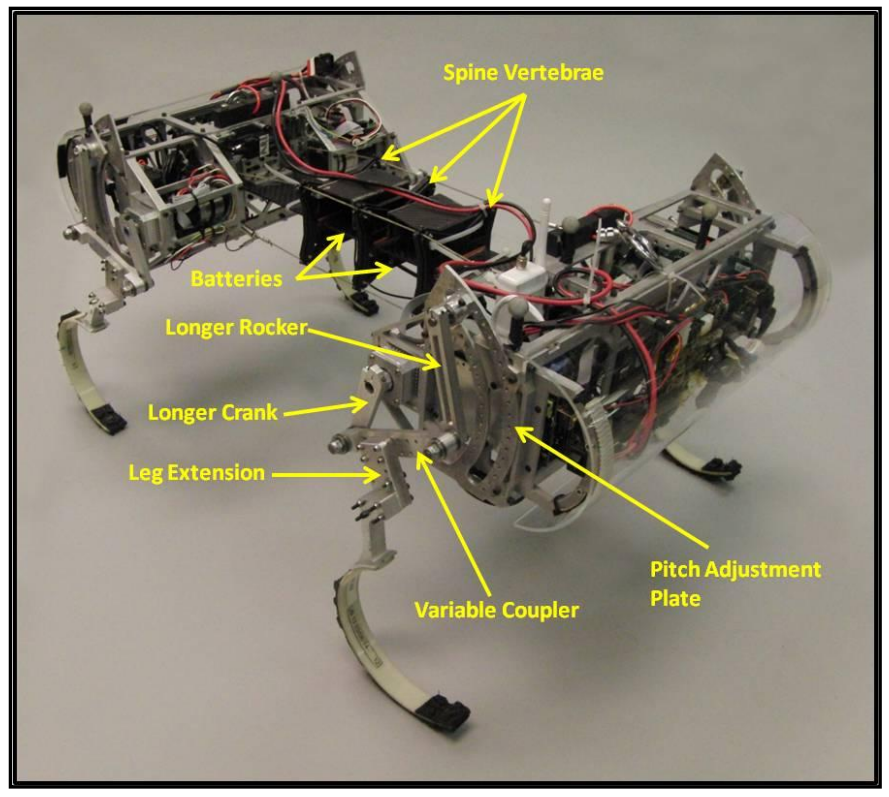

Figure 1: Canid with New Spine and Leg Design Upgrades

\subsubsection{Compliant Legs for High Speed Running}

Canid's simple, compliant legs inherit from the original RHex design a C-shaped woven glass resin composition lined on the outer rim with a rubber tire tread to enhance traction and reduce high frequency impact vibrations [43], [35], [37]. Again, following the RHex model [43], [45], each of Canid's legs is actuated by a single independent motor. In RHex, the C-shaped leg is mounted directly to the motor drive shaft providing a circular toe path. In contrast, following the RiSE example [46], Canid replaces this direct drive with a four bar linkage to enable a high-powered thrust during takeoff followed by a rapid leg recirculation during flight.

The four bar mechanism provides a non-circular toe path and variable transmission better suited for quadrupedal bounding without the use of additional actuators. Figure 2 displays the toe path generated by this linkage in the absence of spine deflection. The rocker shown in Figure 1 is connected to the upper portion the pitch adjustment plate, causing the toe path in Figure 2 to travel approximately parallel to the ground during liftoff and touchdown. This allows Canid to put more of its leg actuator output energy into increasing its forward kinetic energy as opposed to vertical kinetic energy for rapid acceleration and (eventually) high speed running. The depicted temporal parameterization of the toe path shows that the four bar mechanism provides a variable transmission roughly consisting of high torque and low speed during ground contact (the lower portion of the toe path), and high speed and low torque during the recirculation of the legs (the upper portion of the toe path).

Early experiments marred by extensive rear toe stubbing during leg recirculation revealed the need for higher ground clearance, achieved in the next design iteration by lengthening the crank and rocker links using leg extension spacers installed between the coupler and leg mount shown in Figure 1. By creating a longer moment arm for lift off, this adjustment to the four-bar linkage indeed enabled greater clearance heights during flight, and also appeared to 
increase the stride length. Further design iterations of this linkage over the past year have strengthened the components and upgraded the bearings in consequence of bending damage incurred by side loading of the highly energetic body.

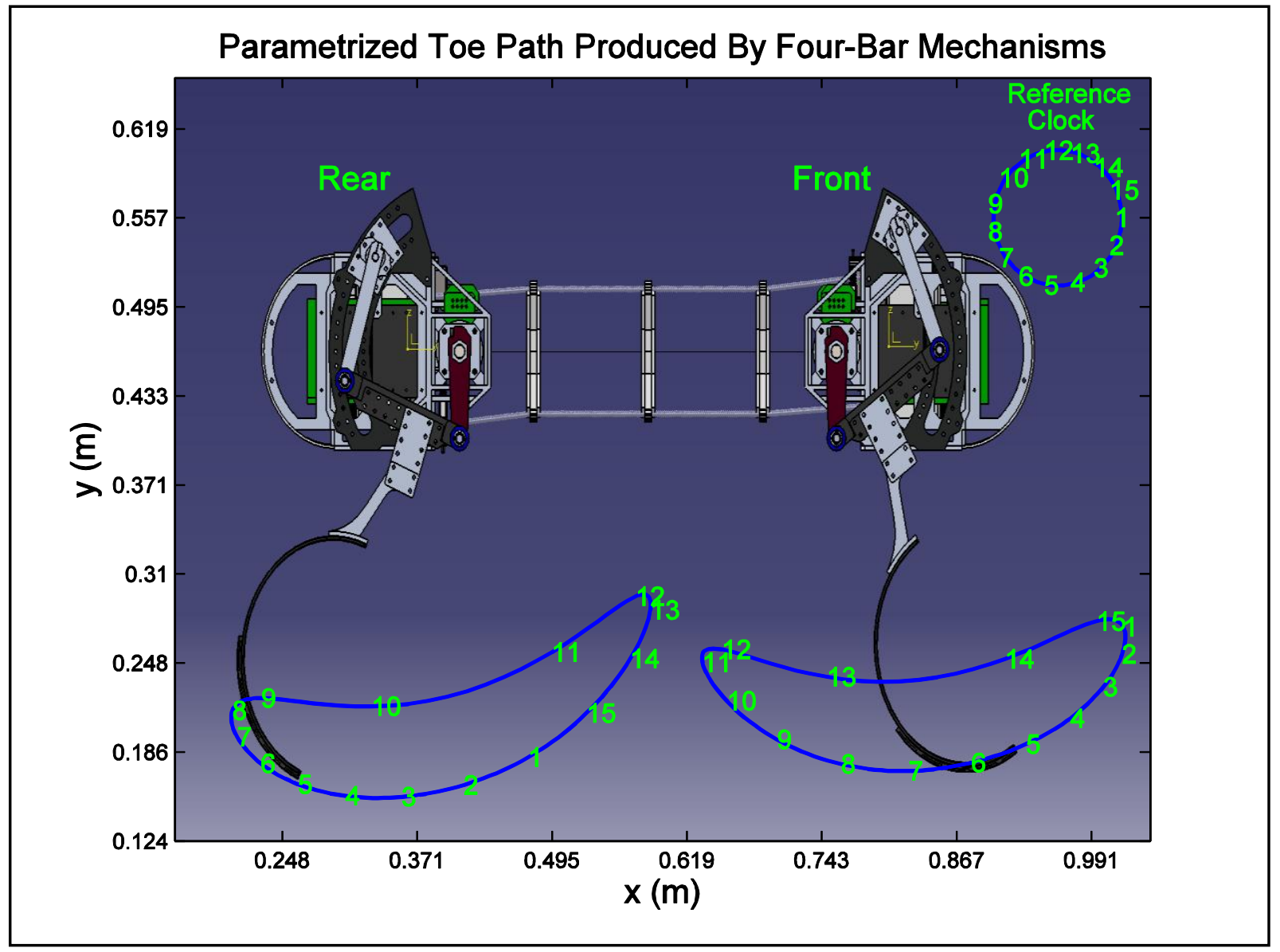

Figure 2: Canid Toe paths for current design. Spine is not flexed and parallel to ground.

\subsubsection{Hardware and Electronics}

While Canid's body exhibits the two major differences in morphology detailed above (its spine and its four-bar driven legs) it is in almost every other regard identical to XRHex and XRL at a systems level, comprised of identical components, incorporating the same actuators, electronics, and software components by virtue of the "laboratory on legs" modular design [20]. At the heart of this common system design, lies a single main central processor (CPU) a PC/104+, $1.6 \mathrm{GHz}$, with $2 \mathrm{~GB}$ of DDR2-DRAM from Advanced Digital Logic that communicates through an Ubiquiti PicoStation M2-HP wireless-N access point to a laptop where it will receive its initial commands. Energy is supplied by two sets of two Thunder Power LiPo 5-cell/5S battery packs, (reduced to one set in the most recent bounding experiments to reduce weight). Initially, located inside the front shoulders and rear hip chassis structure, recent bounding experiments have been conducted with one of these packs removed as a weight reduction measure, and the other relocated to the spine to rebalance the consequently disturbed weight distribution. This power is distributed by custom-built power management board which connects to the COTS Advanced Motion Controls AMC motor controller via a custom interface "double board" [20]. These custom built boards, developed by UPenn GRASP lab researchers, and are designed to communicate a wide range of sensory and control data relating to the motor controllers and batteries to the central computer via USB. The front legs are driven by Maxon flat motor EC-45 with a 28:1 Maxon gearbox. The rear legs use the Maxon ECi-40 pencil motors with a 53:1 Maxon gearbox. The spine is actuated by a Maxon EC-45 with a 28:1 gearbox. Custom built encoder boards also designed by UPenn GRASP lab researchers are used on the front and spine motors.

The front and rear body segments of Canid are equipped with MicroStrain ${ }^{\mathrm{TM}}$ Inertial Measurement Units (IMU) to measure the pitch, roll and yaw of each body relative to the global reference frame. This information potentially will 
be used in future validation and statistical verification of simulation models, and control functions which will attempt to estimate the state of the spine and consequentially the orientation of the two bodies relative to each. A summary of the part list for Canid is included in Table 1.

Table 1: Canid Part List

\begin{tabular}{|c|c|c|c|}
\hline & Description & Manufacturer & Part \# \\
\hline 1 & $\begin{array}{l}\text { - CPU, 8GB on board flash, Intel Atom PC/104+ CPU Board with (Z530) } 1.6 \mathrm{GHz}, \mathrm{Up} \\
\text { to 2GB DDR2-DRAM } \\
\text { - Passive Low Profile Heat sink for CPU } \\
\text { - DDR2 996129, 2GB, 667MHz, } 200 \text { pin for CPU board } \\
\text { - Cable set for CPU board }\end{array}$ & $\begin{array}{l}\text { Advanced Digital } \\
\text { Logic }\end{array}$ & $\begin{array}{l}\text { (ADLS15PC-168) } \\
\text { (ADLS15PC- } \\
\text { PASSIVELP) } \\
\text { (DDR2-DRAM 2GB- } \\
\text { EX (H) 996129) } \\
\text { (ADLS15PC-CK) }\end{array}$ \\
\hline 2 & $\begin{array}{l}\text { Motor Controller Boards - DZRALTE-020L080x, DP Drive, RS232/485, } 20 \text { ADC, } 80 \\
\text { VDC }\end{array}$ & $\mathrm{AMC}$ & DZRALTE-020L080x \\
\hline 3 & $\begin{array}{l}\text { UPenn Power Management Boards - performs battery protection and monitoring, } \\
\text { voltage regulation, and power distribution }\end{array}$ & UPenn & Custom \\
\hline 4 & $\begin{array}{l}\text { UPenn Double AMC interface boards - provides communication between (2) AMC's } \\
\text { and Canid's CPU, \& connection for external USB sensors }\end{array}$ & UPenn & Custom \\
\hline 5 & IMU, 3DM-GX3-25 (non-OEM) & MicroStrain & 3DM-GX3-25 \\
\hline 6 & IMU, 3DM-GX3-35 & MicroStrain & 3DM-GX3-35 \\
\hline 7 & $\begin{array}{l}\text { Batteries, LiPo, } 5 \text {-Cell } / 5 \mathrm{~S} \text { battery Pack, } 2250 \mathrm{mAh}, 18.5 \mathrm{~V} \\
\text { (4 batteries are used on Canid initially now only } 2 \text { batteries are used) }\end{array}$ & Thunder Power RC & TP2250-5SP30 \\
\hline 8 & Wireless Access Point, PicoStation M2-HP, 2.4GHz hi power $801.11 \mathrm{~N}$ & Ubiquiti & PicoStation M2-HP \\
\hline 9 & $\begin{array}{l}\text { Spine Motor (EC45), } 42.8 \mathrm{~mm} \text { dia., flat brushless, } 50 \text { watt } \\
\text { Gearbox }(28: 1), \text { Efficiency }=75 \% \\
\text { Encoder }(1024 \text { counts per turn) } \\
(\text { plus additional gear reduction) }=(40 / 32 /(0.01615 * 2 * \text { pi }))\end{array}$ & $\begin{array}{l}\text { Maxon } \\
\text { Maxon } \\
\text { UPenn } \\
\text { AddGear+Pulley }\end{array}$ & $\begin{array}{l}251601 \\
326662 \\
\text { Custom } \\
\text { Custom }\end{array}$ \\
\hline 10 & $\begin{array}{l}\text { Front Motor (EC-45), } 42.8 \mathrm{~mm} \text { dia., flat brushless, } 70 \text { watt, } \\
\text { Gearbox }(28: 1), \text { Efficiency }=75 \% \\
\text { Encoder }(1024 \text { counts per turn })\end{array}$ & $\begin{array}{l}\text { Maxon } \\
\text { Maxon } \\
\text { UPenn }\end{array}$ & $\begin{array}{l}397172 \\
326662 \\
\text { Custom }\end{array}$ \\
\hline 11 & $\begin{array}{l}\text { Rear Motor }(\mathrm{ECi}-40), 40 \mathrm{~mm} \text { dia., pencil brushless, } 50 \text { watt } \\
\text { Gearbox }(53: 1) \text {, Efficiency }=70 \% \\
\text { Encoder }(2048 \text { counts per turn) }\end{array}$ & $\begin{array}{l}\text { Maxon } \\
\text { Maxon } \\
\text { Maxon }\end{array}$ & $\begin{array}{l}313320 \\
166939 \\
225787\end{array}$ \\
\hline 12 & Carbon Fiber Spine, twill 11 oz, (7 plies, epoxy resin) & $\begin{array}{l}\text { US Composites / } \\
\text { Fab. by UPenn }\end{array}$ & ---- \\
\hline 13 & $\begin{array}{l}\text { Composite Legs: S-2 Structural Fiberglass- } 6781 \text { weave pre-impregnated with } 33 \% \\
\text { MTM28-medium temp molding epoxy resin, legs are } 11 \text { layers thick with layer structure } \\
=(-45,0,+45,0,-45,0,+45,0,-45,0,+45)\end{array}$ & $\begin{array}{l}\text { Advanced } \\
\text { Composite Group } \\
\text { Fab. by UPenn }\end{array}$ & $\begin{array}{l}\text { MTM28/6781 S-2 Glass } \\
33 \% \text { epoxy resin } \\
\text { content, } 50 \text { " wide roll. }\end{array}$ \\
\hline
\end{tabular}

\section{EXPERIMENTS}

\subsection{Spine Characterization}

Characterizing the energy stored in Canid's spine is a necessary step in assembling the methodology to measure the most significant components of Canid's body energy while bounding. The method of characterization while neglecting the complexity of the spine's composite mechanics, arguably approximates its dominant capacity for storing energy by postulating a lumped torsional spring across a one degree of freedom revolute joint and allows for proprioceptive estimation of that elastic energy using the surrogate of angular position in Canid's motors.

\subsubsection{Setup and Experiments}

Canid's spine was characterized using a duplicate mechanism constructed identically to that used on the robot. The entire spine assembly with its actuating cable frame intact was loaded with successively increasing known weights to measure the resulting compression induced by forces typically experienced in Canid's bounding. The energy stored in the spine as a function of this controlled gravitational loading was calibrated in units of actuator cable-length, resulting in an intrinsic, proprioceptive measure suitable for sensing and recording during real time operation. The details of this process are given below. 


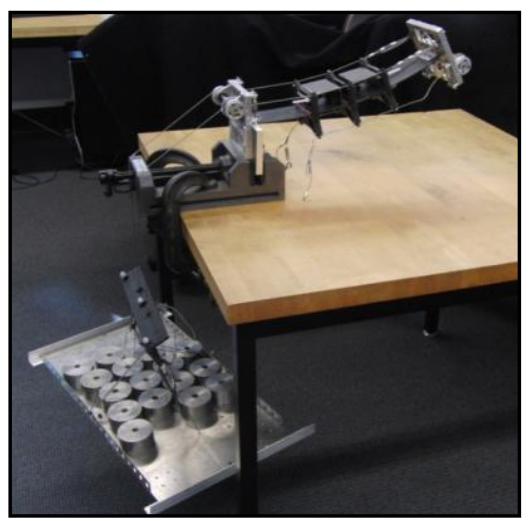

Figure 3: Weights were suspended from the spine cables imposing a gravitational load whose resulting flexion could be measured in the same units of cable lengths as accessible to actuators during physical operation. The change in potential energy of the system was calculated by multiplying the weight of the platform by its Vicon measured displacement giving the relationship between cable flexion displacement and potential energy stored.

Figure 3 shows the isolated spine assembly under load: carbon fiber leaf, vertebrae, cables, and front and rear spine connector plates. The rear plate was rigidly anchored to a table and the top spine cables were attached to a platform that was allowed dangle below the table. Weights were added to the platform to mimic the force applied by Canid's spine motors, putting tension on the top spine cables, causing the assembly to bend upwards. Placing increasingly heavy weights on the metal platform caused the cable displacement to monotonically increase as energy was loaded into the spine. This cable displacement was determined by calculating the displacement of the weight platform using a Vicon motion capture system. The corresponding motor position is in turn calculated from the gear ratio of the spine pulley system.

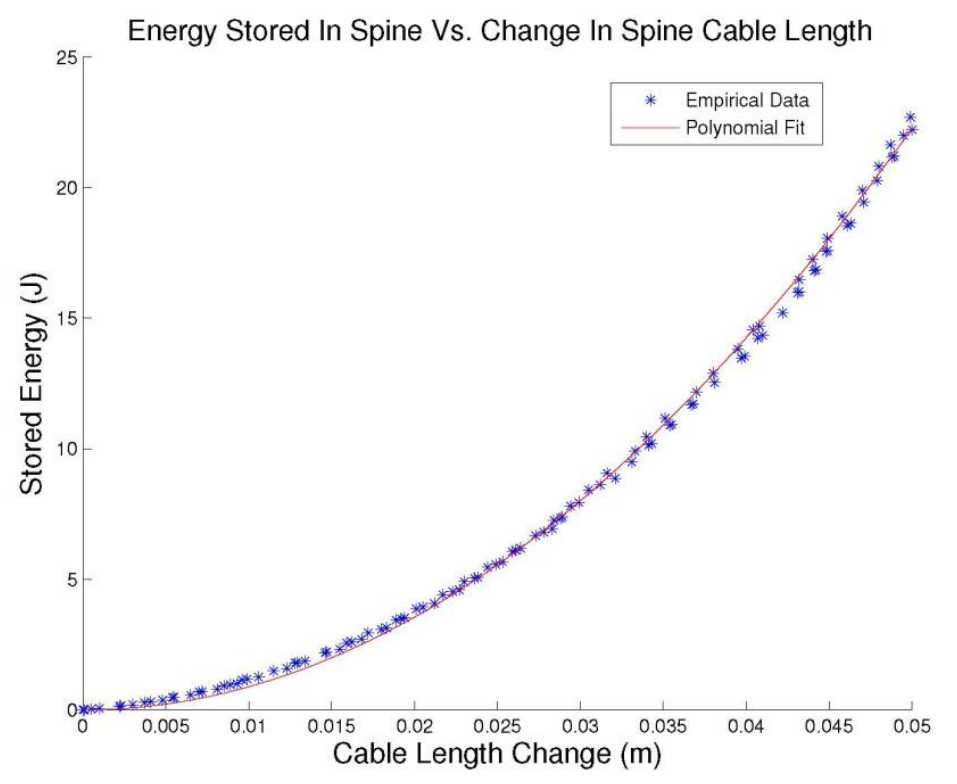

Figure 4: Representation of the spine leaf spring potential energy function via a convex polynomial fit with a residual (rootmean-squared) error of $0.30 \mathrm{~J}$. This polynomial is used to proprioceptively calculate the spine energy onboard Canid during a run based on the spine motors' angular position, by multiplying the cable length by the appropriate gear ratio of the pulley system connecting the spine cables to the motors.

\subsubsection{Results}

The change in potential energy of the system is calculated by multiplying the weight of the platform by its displacement. Since we observe that the cables are far stiffer than the spine mechanism, we ignore their intrinsic 
compliance and assume that the change in gravitational potential energy of the platform is equal to the spring potential energy stored in the spine bending. A convex polynomial was fit to the measured cable deflections of various spine energies, giving the relationship between cable displacement and stored spine potential energy shown in Figure 4. Multiplying by the appropriate gear ratio of the pulley system attaching the spine motor to the spine cables gives the potential energy stored in the spine in units of motor output shaft position. Implicit in this method is the assumption that the cables are taut during Canid's bounding, which was verified by close inspection of high-speed video data taken during typical bounding episodes. Since we would prefer our measurement errors to under-estimate rather than overestimate true physical energy during online operation, it is reassuring to note that if the cables were not taut then this method would only underestimate the stored spine energy.

\subsection{Leg Characterization}

\subsubsection{Setup and Experiments}

The C-legs were characterized using a leg testing rig to measure various leg deflections for approximately radial loads and varying touchdown points along the perimeter of the leg as shown in Figure 5. For each touchdown point along the leg's circumference, the leg acts roughly as a linear spring with a $4.27 \mathrm{~N}$ and $4.49 \mathrm{~N}$ root-mean-squared-error in the front and rear legs (respectively) as shown by data collected in Figure 6, resulting in a noise floor of 4.8\% and 5\% for the front and rear legs (respectively). This empirically observed composite linear spring is in fact the result of 2 series springs: the elastic compliance from the fiberglass composite and the rubber tread. The spring constant from the fiberglass leg composite varies continuously along the circumference of the leg while the rubber tread spring constant remains roughly the same regardless of touchdown point. These linear spring constants were measured for 3 touchdown points on Canid's legs and interpolated to estimate the spring constants for intermediate touchdown points. The front legs were chosen to be more compliant than the rear legs, but each pair of legs in the front and rear were manufactured to have approximately the same material properties. Thus, it was only necessary to test 2 out of the 4 legs ( 1 front leg and 1 rear leg).

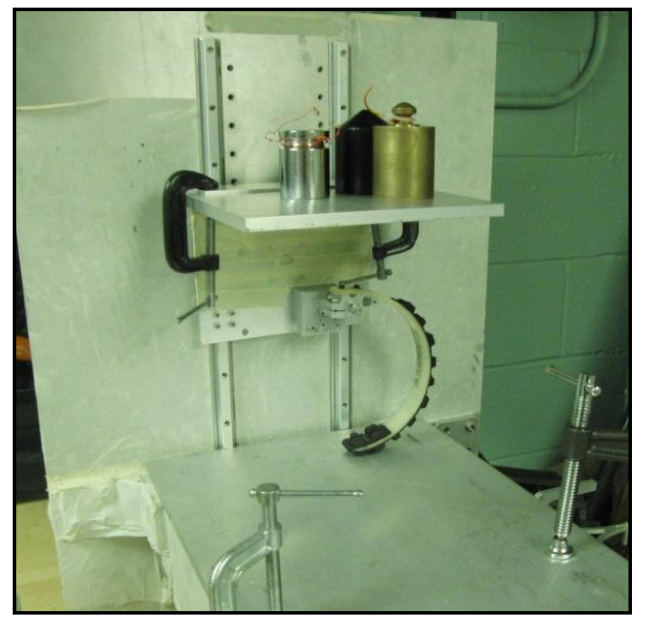

Figure 5: Leg deflection for approximately radial loads was measured on a rig for various touchdown points along the perimeter of the leg.

\subsubsection{Results}

When Canid is running, the leg touchdown points and deflection magnitudes are measured as follows. Canid's body pose is calculated from the robot's position and orientation as measured under Vicon. The robot's estimated toe position is calculated from its body pose and leg motor shaft angle assuming an uncompressed C-leg. If this toe location is kinematically unfeasible (i.e. if some part of the leg is below the ground), then we know there must be ground contact. We assume circular leg deflection (an approximation), so that we can estimate the ground contact point of the leg by fitting the largest circle to the leg that is kinematically feasible to the end of the four-bar mechanism the radius of which will be less than that of the uncompressed leg such that the circle lies tangent to the ground. The point where the circle intersects the ground is the estimated ground-contact point of the leg. The estimated leg deflection is given by the difference between the uncompressed leg diameter and the diameter of the fit circle. 


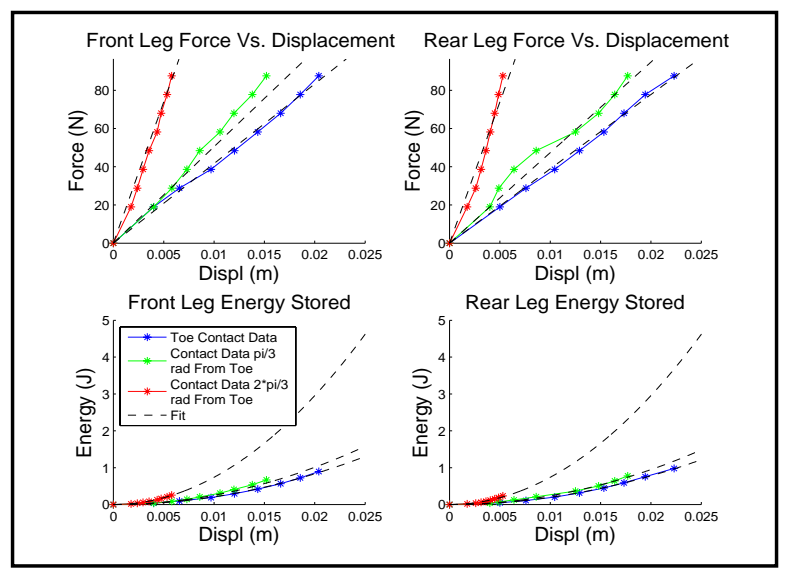

Figure 6: Results of leg testing. The top figures show that for a fixed touchdown position along the perimeter of the legs, under radial loading the legs act as approximately linear springs with root-mean-squared errors of $4.27 \mathrm{~N}$ and $4.49 \mathrm{~N}$ for the front and rear legs, respectively. The bottom plots give the potential energy stored in the legs for various touchdown points.

This method is inspired by [2] but our implementation is nevertheless a crude approximation of the true leg energy storage because significant torsional loads from the motors are present but unaccounted for. This motor torsion hasn't been explicitly addressed to our knowledge in the literature but it is reasonable to expect since the motors generate significant torques on the legs after being geared down by suitable transmissions. Hence, we only use this characterization to make very rough qualitative statements about the leg energy storage since the characterization is an obvious approximation. To date, over their decade long discussion in the literature, no C-leg models of which we are aware [2], [3], [19], [38] can account for both variable touchdowns on the leg as well as torsional loads from the motor and ground forces.

\subsection{Preliminary Canid Bounding Data}

Table 2: Canid Parameters and Preliminary Operational Statistics

\begin{tabular}{|l|l|}
\hline Mechanical Design Parameters & Canid \\
\hline Mass (kg) & 11.3 \\
Body Length (m) & 0.78 \\
Front Hip to Rear Hip Length (m) & 0.39 \\
Maximum Hip Height (m) & 0.288 \\
\hline Electrical Design Parameters and Operating Statistics & \\
\hline Maximum Allowed Motor Voltage (V) & 34 \\
\hline Listed Motor Power (W) & \\
\hline - Front & 70 \\
$-\quad$ Middle & 50 \\
$-\quad$ Rear & 50 \\
\hline Mean Observed Electrical Power (W) & \\
\hline - Front (2 motors combined) & $35 \pm 52$ \\
$-\quad$ Middle (2 motors combined) & $115 \pm 98$ \\
$-\quad$ Total (6 motors combined & $219 \pm 205$ \\
\hline Robot Body Operating Statistics & $368 \pm 271$ \\
\hline Mean Stride Length (m) & \\
Mean Stride Frequency (Hz) & $0.82 \pm 0.09$ \\
Mean Duty Factor & $1.31 \pm 0.11$ \\
Mean Steady-State Forward Vel. (m/s) & $0.55 \pm 0.04$ \\
(body-lengths / sec) & $1.38 \pm 0.19$ \\
Maximum Forward Velocity (m/s) & $1.97 \pm 0.27$ \\
(body-lengths / sec) & 2.18 \\
\hline
\end{tabular}


This section documents measurements collected during Canid's very first repeatable bounding experiments, comprising a set of 3 runs under the Vicon with 3 strides per run. The data from these runs is summarized in Table 2, indicating that Canid's present open loop set-point driven bounding achieves a mean speed of roughly 2 bodylengths per second, with bursts of speed approaching 3 bodylengths per second. We now describe the qualitative features of Canid's operation through the various phases of its hybrid dynamical operation, presenting an in-depth breakdown of the energetics of a typical Canid stride. We then present the energetic data for all of the runs to provide the reader with a more detailed and quantitative account of the robot's behavior.

\subsubsection{Hybrid Dynamics}

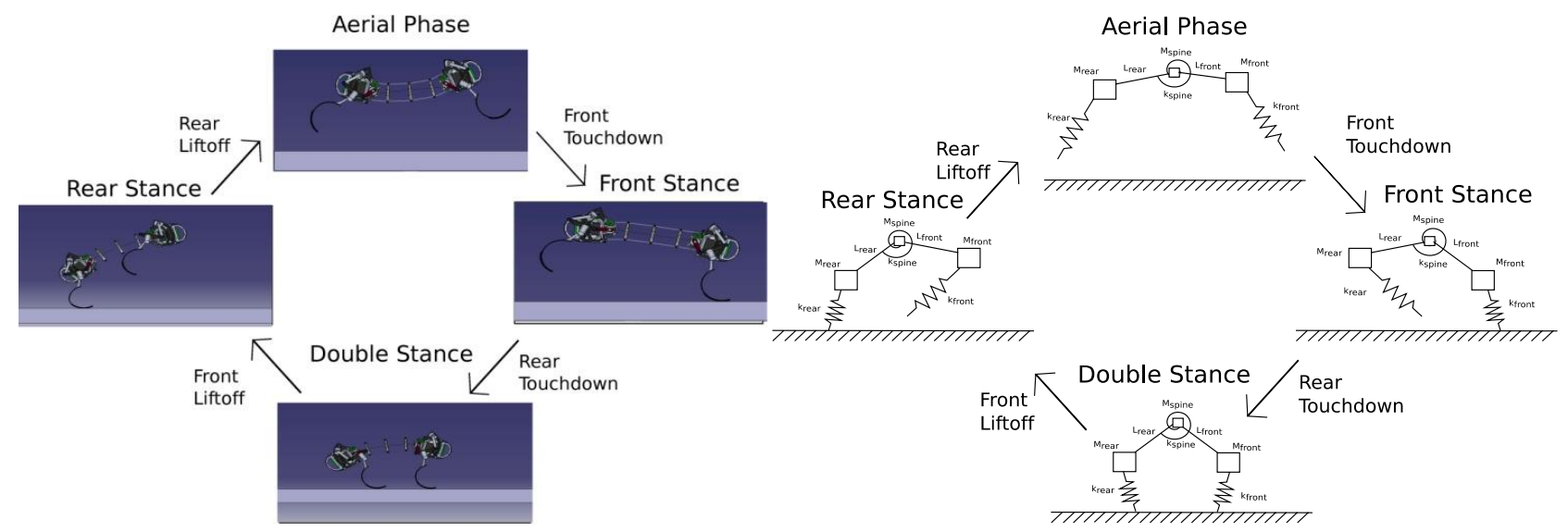

Figure 7: Hybrid dynamics of Canid are illustrated in the left graphic, while the right portrays a greatly simplified, spring coupled point mass approximate model used to define "body energy" and depicted in each of its corresponding hybrid states.

Canid's current bounding behavior can be described as a hybrid dynamical system with 4 distinct hybrid states, its "phases," shown in Figure 7: aerial phase (where no legs touch the ground), front stance (where the front legs are in contact with the ground), rear stance (where the rear legs are in contact with the ground), and double stance (where both front and rear legs are in contact with the ground).

We define the body energy in each of these hybrid states in terms of the greatly simplified approximate mechanical model depicted in the right hand graphic of Figure 7. This approximation estimates Canid's kinetic energy, gravitational potential, and elastic spring potential energy (stored in both the elastic spine as well as the leg compliance) by replacing its physical rigid bodies and links with three point masses and lumping all compliant elements into simple one degree of freedom revolute (the spine) and prismatic (the legs) joints. The instantaneous locus of each of these point masses is computed from Vicon measurements of calibrated markers on its rear and front hips and its spine-located battery pack, respectively. The gravitational potential energy for each body segment is given by multiplying the weight of the rear, front and spine components by the height above the ground of their point mass representatives. Similarly, the kinetic energy is given by multiplying one half their corresponding masses by the magnitude of the squared velocities of their respective point mass representatives. Clearly, our neglect of any rigid body features ignores all rotational kinetic energy and our calculation is an unequivocal underestimate of true kinetic energy (although the discrepancy is likely not very great because high-speed video suggests that the vast majority of Canid's kinetic energy is not rotational). The overall gravitational potential energy and kinetic energy are then calculated by summing the individual respective energies of the point mass body segment representatives. The methods of calculating the potential energy stored in the spine and legs are given in Sections 3.1 and 3.2. We believe that these also underestimate the physical energy stored and released by the various compliant elements in the legs and spine, but the arguments supporting this view seem quite a bit more complex, and lie beyond the scope of the present paper.

\subsubsection{A Typical Canid Leap}

Even disregarding our central focus on energy flows, the scalar components measured and summed to compute body energy as just defined offer a very convenient means of dimension reduction, providing an intuitively informative quantitative summary of the high degree of freedom trajectories exhibited by this mechanism's complex dynamics (observe that even the simplified representation in the right hand graphic of Figure 7 entails a state space of dimension 8 
in its aerial phase). Thus, to give the reader a sense of a typical Canid leap, we closely examine the body energy plots given in Figure 8 representing the first leap of a run under Vicon.

Canid begins the run stationary in rear stance, crouched at rest with its rear legs on the ground and flexing its spine to store 17J of spring potential energy. At 9.93 seconds, Canid begins to push off with its rear legs and un-flex its spine. The spine motors output 41J of mechanical energy on liftoff working in parallel with the elastic spine compliance while the rear legs push off and do 47J of mechanical output energy. The front legs begin to slowly recirculate to prepare for their eventual ground contact, only spending 12J of mechanical output energy. By 10.27 sec Canid is in flight and has nearly $70 \mathrm{~J}$ of mechanical body energy, roughly $20 \mathrm{~J}$ as kinetic energy and 50J as gravitational potential energy.

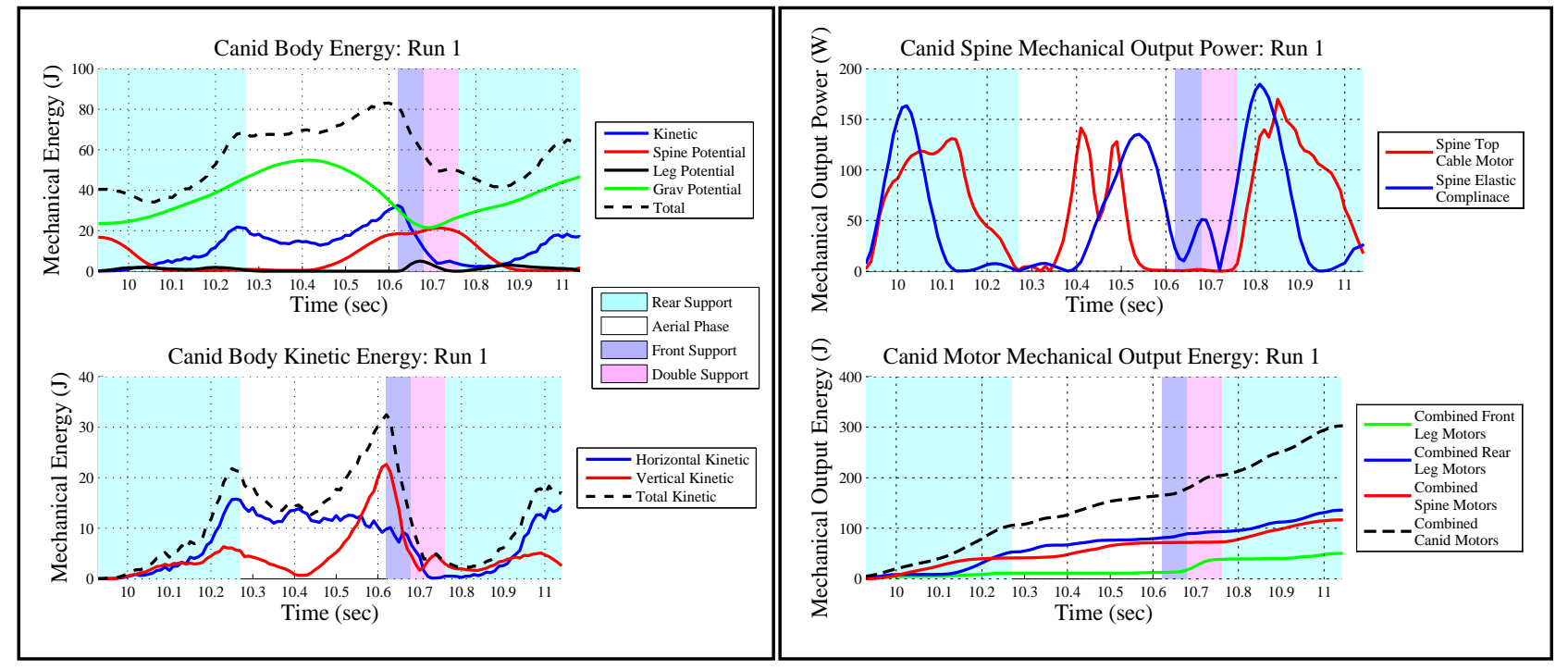

Figure 8: Mechanical energy and power of Canid during a typical run.

Note that the elastic spine compliance power profile is very different from the power profile of the spine motors. The spine spring is able to output a peak of slightly over $160 \mathrm{~W}$ of power very early in the leap, while the spine motor controlling the top cables (responsible for flinging the front mass forward) operates only at roughly $120 \mathrm{~W}$ but for over twice as long in duration.

The first aerial phase lasts from $10.27 \mathrm{sec}$ to $10.62 \mathrm{sec}$. During this time, Canid recirculates its legs in anticipation of touchdown, outputting $30 \mathrm{~J}$ in its rear leg motors and only $1 \mathrm{~J}$ in its front legs, which remain largely stationary during Canid's flight. Canid also begins to flex its spine, expending $30.5 \mathrm{~J}$ of mechanical work with its spine actuators to store $18 \mathrm{~J}$ of spine spring potential energy.

Front support begins at 10.62 with the touchdown of the front legs. Kinetic energy begins to decrease as Canid converts vertical kinetic energy into spine spring potential energy in the legs and into flexing the spine. Canid's front four-bar mechanisms land in a singular condition, allowing for energy transfer into leg spring potential energy without significant actuator work. The front leg motors only begin to output significant power during the second half of the front-stance after good ground contact has been achieved.

Double support begins at 10.72 seconds when the rear legs touchdown. Due to open loop disturbances, Canid's kinetic energy drops to 3J. Uncontrolled roll perturbations in the rear legs are suspected to be the main culprit for this decrease as discussed in [14]. Mechanical body energy continues to be used to flex the spine, which allows Canid to store $10 \mathrm{~J}$ more energy in the spine elastic compliance than is possible through just using the spine actuators alone due to actuator torque limits ${ }^{3}$, allowing Canid to store a total of $21 \mathrm{~J}$ of spring potential energy. This represents a large fraction

\footnotetext{
${ }^{3}$ Due to a complicated current limiting scheme, we cannot report a simple maximum allowed motor current for Canid. However, no motors besides Canid's spine motors reached their current limit during the trials. Canid's top spine-cable motor was limited to 8A while its bottom spine-cable motor was limited to 4A for thermal considerations. These differing current limits are necessitated by an
} 
of Canid's body energy, equal to roughly $31 \%$ of Canid's body energy at the time of liftoff at the beginning of the previous aerial phase.

Canid begins its 2nd leap in rear stance with only $3 \mathrm{~J}$ of kinetic energy (due to open-loop inefficiencies) but with $21 \mathrm{~J}$ of energy stored in its spine elastic compliance. As discussed in [14], these open-loop inefficiencies induced roll disturbances that prevent Canid from achieving good rear leg ground contact when the spine is releasing its spring potential energy. Thus, we suspect that much of this elastic spine energy release is wasted.

The 2nd stride is very similar to the 1st as shown in Figure 9 for all the runs aside from the varied kinetic energy in rear stance, the amount of spine energy stored at the beginning of rear stance, and the efficiency of spine spring potential energy transfer into mechanical body energy during rear stance due to open-loop disturbances. The 3rd stride is more varied, which is owed to the chaotic nature of the open-loop inefficiencies.
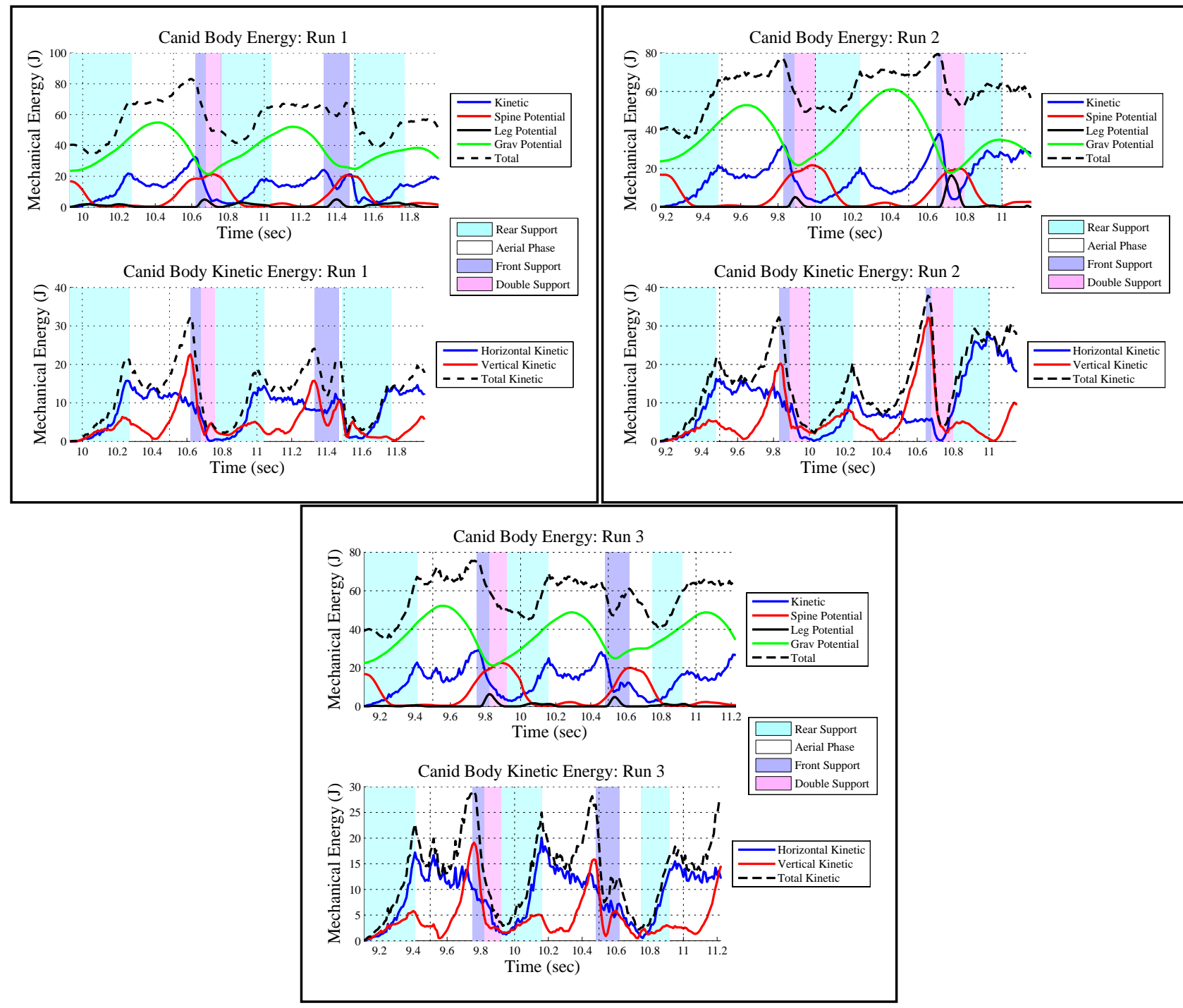

Figure 9: Body energy of Canid during runs showing Canid's average body energy was 58J.

admittedly inefficient startup procedure that heats up the bottom spine-cable motor before the Canid's first leap. This startup procedure will be improved in later experiments. 
Figure 9 shows the body energy of Canid during all of its runs. The mean body energy was 58J. The runs have similar energetic profiles up until the 2 nd front stance when open-loop disturbances show noticeable energetic variations between the runs.

\section{CONCLUSION AND FUTURE DIRECTIONS}

We have presented a detailed discussion of the relatively few design innovations needed to transform a rigid bodied hexapod, the XRL update [20] of the decade old RHex robot generalist [43], into a much more energetic but commensurately more specialized flexible spine bounding robot, Canid. We have introduced what we believe to be a new, empirically accessible scalar quantification of a highly dynamical, complex mechanism that we term "body energy," and describe the instrumentation and methodology developed to measure it during our robot's real time operation. Finally, we use that methodology to document what we believe to be the first bounding behavior of a freestanding, power-autonomous, flexible spine actuated, legged machine.

Because Canid's locomotion is presently driven by an open loop succession of pre-designed actuator set points, it would not be fair to say that this preliminary behavior represents a true bounding gait in the steady state sense that term connotes. Indeed, under the present open loop regime, disturbances introduced by uncoordinated rear leg touchdowns excite oscillations in the highly elastic spine whose unsensed (hence crudely achieved) dissipation incurs nearly complete extinction of forward momentum at the completion of each stride. For purposes of this paper, we have found it sufficient to report in detail on 3 successful runs of Canid's leaping over the course of 3 successive strides each. Because this succession of leaps is so repeatable (both within the first two leaps of a run as well as between runs), we find it appropriate to use the term open loop bounding to describe the behavior. The restriction to 3 strides was chosen because it fits easily within the space constraints imposed by our Vicon facility, is long enough to gather the data we need, and can be readily accomplished in a few days of gait tuning following small changes to the machine. Since Canid does not accelerate significantly between strides due to the uncoordinated (open-loop) rear touchdowns and the clumsy recovery, we are convinced that more strides per run would yield quantitative results very similar to those documented here. Work now in progress focuses on adding enough proprioceptive and vestibular feedback to secure true, stable, steady state bounding.

Although stable bounding is intrinsically interesting, we are at least as strongly motivated in this work by the hope to begin to understand the influence of morphology and systems level design on emergent robot capabilities. Thus, in new work presently under review [15] we have compared in great detail the energetics of Canid's bounding as introduced here, with the highest energy gait, the pronk, achievable by its rigid-bodied cousin, XRL. We do indeed find that Canid operates at nearly double the body energy, as does the pronking hexapod and with no greater mechanical specific resistance notwithstanding the presently highly wasteful nature of these open loop bounds. It seems nearly certain that closed-loop stabilized versions of Canid's bounding will greatly accentuate its energetic advantage with superior efficiency of locomotion as well.

Future work will be required to reveal just how specialized Canid is relative to the ubiquitously useful decade old locomotion generalist, RHex. In particular, it remains to be seen whether or not we can harness Canid's much higher energetic mode of operation into more nimble transitional behaviors than XRL can achieve, or, perhaps, to leverage superior rejection of severe disturbances from broken, complex terrain. More generally, given a fixed set of components and power budget, we would like to better understand what is required to achieve greater agility in legged robot design.

\section{ACKNOWLEDGEMENTS}

The authors thank members of Kod*lab within Penn's GRASP lab for many helpful discussions and a significant amount of assistance in running experiments. This work is supported by the National Science Foundation Graduate Research Fellowship under Grant Number DGE-0822, and by the Army Research Laboratory under Cooperative Agreement Number W911NF-10-2-0016.

\section{REFERENCES}

[1] Ananthanarayanan, A., Azadi, M., and Kim, S., "Towards a Bio-Inspired Leg Design for High-Speed Running," Bioinspiration \& Biomimetics, Vol. 7, (2012). 
[2] Ankarali, M., Sayginer, E., Yazicioglu, Y., Saranli, A., and Saranli, U., “A Dynamic Model of Running with a HalfCircular Compliant Leg," Proc. of the $15^{\text {th }}$ Int. Conf. on Climbing and Walking Robots and the Support Technologies for Mobile Machines, vol. 23, pp. 26, (2012).

[3] Aydin, Y. O., Galloway, K. C., Yazicioglu, Y., and Koditschek, D. E., "Modeling the Compliance of a Variable Stiffness C-Shaped Leg Using Castigliano's Theorem," Proc. of the ASME Design Engineering Technical Conf., Vol. 2, pp. 705-713, (2010).

[4] Berns, K., Ilg, W., Deck, M., and Dillmann, R., "The Mammalian-Like Quadrupedal Walking Machine BISAM," Proc. AMC $5^{\text {th }}$ Int. Workshop on Advanced Motion Control, pp. 429-433, Coimbra, Portugal, (29 Jun.-1 Jul. 1998).

[5] Biewener, A. A., and Taylor, C. R., "Bone Strain: A Determinant of Gait and Speed," J Exp. Biol. 123, pp. 383-400, (1986).

[6] Boston Dynamics, http://www.bostondynamics.com

[7] Breithaupt, R., Dahnke, J., Zahedi, K., Hertzberg, J., and Pasemann, F., "Robo-Salamander - An Approach for the Benefit of Both Robotics and Biology," Proc. of the $5^{\text {th }}$ Int. Conf. on Climbing and Walking Robots (CLAWAR), p. 55, (Sept. 2002).

[8] Campbell, D., Buehler, M., "Preliminary Bounding Experiments in a Dynamic Hexapod," Experimental Robotics VIII, pp. 612-621, (2003).

[9] Cao, Q., Poulakakis, I., "Passive Quadrupedal Bounding with a Segmented Flexible Torso," IEEE/RSJ Int. Conf. on Intelligent Robot and Systems, Vilamoura, Algarve, Portugal, (7-12 Oct. 2012).

[10] Crespi, A., "From Swimming to Walking with a Salamander Robot Driven by a Spinal Cord Model," Science, vol. 315(5817):1416, (2007).

[11] Culha, U., "An Actuated Flexible Spinal Mechanism for a Bounding Quadrupedal Robot," Thesis for Master of Science in Computer Engineering at Bilkent University, (Jan. 2012).

[12] Culha, U., Saranli, U., "Quadrupedal Bounding with an Actuated Spinal Joint," Accepted for Publication in IEEE International Conference on Robotics and Automation, Shanghai, China, (May 2011).

[13] Deng, Q., Wang, S., Xu, W., Mo, J., and Liang, Q., "Quasi Passive Bounding of a Quadruped Model with Articulated Spine," Mechanism and Machine Theory, Vol. 52, pp. 232-242, (2012).

[14]Duperret, J. M., Pusey, J. L., Haynes, G. C., and Koditschek, D. E., "Canid, Pronking XRL Preliminary Comparison Dataset,” Technical Report, available http://kodlab.seas.upenn.edu/Jeff/CanidTR13, U. of Pennsylvania, (2013).

[15] Duperret, J. M., Pusey, J. L., Haynes, G. C., and Koditschek, D. E., "Energetic Benefits of Elastic Spine Actuation for a Quadrupedal Robot," submitted for presentation at IEEE IROS, (2013).

[16] Farve, N. N., "Design of a Low-Mass High-Torque Brushless Motor for Application in Quadruped Robotics," Master of Science in Electrical Engineering and Computer Science at MIT, Master's thesis, (Jun. 2012).

[17] Fischer, M. S., Witte, H., "Legs Evolved Only at the End!," Phil. Trans. R. Soc. A, pp. 185-198, (2007).

[18] Folkertsma, G. A., Kim, Sangbae, Stramigioli, S., "Parallel Stiffness in a Bounding Quadruped with Flexible Spine," IEEE RSJ International Conf on Intelligent Robots and Systems, Vilamoura, Algarve, Portugal, Oct. 7-12, (2012).

[19] Galloway, K. C., Clark, J. E., and Koditschek, D. E., "Variable Stiffness Legs for Robust Efficient , and Stable Dynamic Running," J. of Mechanisms and Robotics, Vol. 5, No. 1, (2013).

[20] Haynes, G. C., Pusey, J. L., Knopf, R., Johnson, A. M., and Koditschek, D. E., "Laboratory on Legs: An Architecture for Adjustable Morphology with Legged Robots," Proc. of the SPIE Defense, Security, and Sensing Conf., in Unmanned Systems Technology XIV, vol. 8387, no. 1, p. 83870W, (2012).

[21] Haynes, G. C., Pusey, J. L., Knopf, R., and Koditschek, D. E., "Dynamic Bounding with a Passive Compliant Spine," Proc. Dynamic Walking Conf., (2012).

[22] Higham, T. E., and Jayne, B. C., "Locomotion of Lizards on Inclines and Perches: Hindlimb Kinematics of an Arboreal Specialist and a Terrestrial Generalist," J. Exp. Biol., Vol. 207, No. 2, pp. 233-248, (Jan. 2004).

[23] Hildebrand, M., "Motions of the Running Cheetah and Horse," J. of Mammalogy, Vol. 40, No. 4, pp. 481-495, (20 Nov. 1959).

[24] Hudson, P. E., Corr, S. A., and Wilson, A. M., "High Speed Galloping in the Cheetah (Acinonyx Jubatus) and the Racing Greyhound (Canis Familiaris): Spatio-Temporal and Kinetic Characteristics," The J. of Expl. Bio., Vol. 215, pp. 2425-2434, (2012).

[25] Hunter, I. W., Hollerbach, J. M., and Ballantyne, J., "A Comparative Analysis of Actuator Technologies for Robotics,” Robotics Review, Vol. 2, pp. 299-342, (1992).

[26] Johnson, A. M., Haynes, G. C., and Koditschek, D. E., "Disturbance Detection, Identification, and Recovery by Gait Transition in Legged Robots," in 2010 IEEE/RSJ International Conference Intelligent Robots and Systems (IROS), pp. 5347-5353, (2010). 
[27] Johnson, A. M., and Koditschek, D. E., “Toward a Vocabulary of Legged Leaping," presented at the IEEE Int. Conf. Rob. Aut. pp. (to appear), (2013).

[28] Kani, M. H. H., Derafshian, M., Bidgoly, H. J., Ahmadabadi, M. N., "Effect of Flexible Spine on Stability of a Passive Quadruped Robot: Experimental Results," IEEE International Conf on Robotics and Biomimetics, Phuket, Thailand, (Dec. 7-11, 2011).

[29] Kim, K. H., Park, S. H., and Lee, Y. J., "Development of Biomimetic Quadruped Walking Robot with 2-DoF Waist Joint," Proc. of SPIE, pp. 60422, (Dec. 2005).

[30] Laffranchi, M., Sumioka, H., Sproewitz, A., Gan, D., Tsagarakis, N. G., “-MARSi Adaptive Modular Architectures for Rich Motor Skills - Compliant Actuators," ICT-248311, D 2.1, (Mar. 2011).

[31] Leeser, K. F., "Locomotion Experiments on a Planar Quadruped Robot with Articulated Spine," Thesis for Masters of Science in Mechanical Engineering at the Massachusetts Institute of Technology, (Feb. 1996)

[32] Lewis, M. A., "Self-Organization of Locomotory Controllers in Robots and Animals," Ph.D. dissertation in Electrical Engineering at University of Southern California, (Aug. 1996).

[33] Lewis, M. A., "Gait Adaptation in a Quadruped Robot," Autonomous Robots, (2002).

[34] McKenzie, J. E., "Design of Robotic Quadruped Legs," Master of Science in Mechanical Engineering at MIT, Master's thesis, (Feb. 2012).

[35] McMordie, D., and Buehler, M., "Towards Pronking with a Hexapod Robot," in Proc. $4^{\text {th }}$ Intl. Conf. on Climbing and Walking Robots, Storming Media, pp. 659-699, (2001).

[36] Moore, E. Z., Campbell, D., Grimminger, F., and Buehler, M., "Reliable Stair Climbing in the Simple Hexapod RHex," in Proc. IEEE Int. Conf. on Robotics and Automation, Vol. 3, pp. 2222-2227, (2002).

[37] Moore, E. Z., "Leg Design and Stair Climbing Control for the RHex Robotic Hexapod," Ph.D. Dissertation, McGill University, (2002).

[38] Ogucu, M., Saranli, A., and Yazicioglu, "Modeling Half-Circular Compliant Legs by Using Simmechanics," Proc. of the $15^{\text {th }}$ Int. Conf. on Climbing and Walking Robots and the Support Technologies for Mobile Machines, Vol. 23, (2012).

[39] Park, S., and Lee, Y.J., "Discontinuous Zigzag Gait Planning of a Quadruped Walking Robot with a Waist-Joint," Advanced Robotics, vol. 21(1), pp. 143-164, (Jan. 2007).

[40] Poulakakis, I., Smith, J. A., Buehler, M., "Modeling and Experiments of Untethered Quadrupedal Running with a Bounding Gait: The Scout II Robot,” Int. J. of Robotics Research, Vol. 24, No. 4, pp. 239-256, (Apr. 2005).

[41] Pouya, S., Khodabakhsh, M., Moeckel, R., and Ijspeert, A., "Role of Spine Compliance and Actuation in the Bounding Performance of Quadruped Robots," $7^{\text {th }}$ Dynamic Walking Conference, USA, (May 2012).

[42] Reisz, R. R., Scott, D., Sues, H. D., Evans, D. C., and Raath, M. A., "Embryos of an Early Jurassic Prosauropod Dinosaur and Their Evolutionary Significance," Science, Vol. 309, No. 5735, pp. 761-764, (Jul 2005).

[43] Saranli, U., Buehler, M., and Koditschek, D. E., "RHex: A Simple and Highly Mobile Hexapod Robot," The Int. J. of Robotics Research, Vol. 20, No. 7, p. 616, (2001).

[44] Seok, S., Wang, A., Otten, D., and Kim, S., "Actuator Design for High Force Proprioceptive Control in Fast Legged Locomotion," IEEE Int. Conf. on Intelligent Robots and Systems, pp. 1970-1975, (2012).

[45] Smith, J. A., "Galloping, Bounding and Wheeled-Leg Modes of Locomotion on Underactuated Quadrupedal Robots," Ph.D. thesis, McGill University, (2006).

[46] Spenko, M. J., Saunders, J. A., Haynes, G. C., Cutkosky, M. R., Rizzi, A. A., Full, R. J., and Koditschek, D. E., "Biologically Inspired Climbing with a Hexapedal Robot," Journal of Field Robotics, Vol. 25, No. 4-5, pp. 223-242, (2008).

[47] Takuma, T., Ikeda, M. and Masuda, T. "Facilitating Multi-Modal Locomotion in a Quadruped Robot Utilizing Passive Oscillation of the Spine Structure,” The 2010 IEEE/RSJ International Conference on Intelligent Robots and Systems, Taipei, Taiwan, (Oct. 18-22, 2010).

[48] Valenzuela, A. K., "Stride-Level Control of Quadrupedal Runners Through Optimal Scaling of Hip-Force Profiles," Master of Science in Mechanical Engineering at MIT, Master's Thesis, (Sept. 2011).

[49]Zhao, Q., Nakajima, K., Sumioka, H., Yu, X., and Pfeifer, R., "Embodiment Enables the Spinal Engine in Quadruped Robot Locomotion,” IEEE RSJ International Conf on Intelligent Robots and Systems, Vilamoura, Algarve, Portugal, (Oct. 7-12, 2012).

[50]Zeglin, G. "The Bow Leg Hopping Robot," CMU-RI-TR-99-33, Doctor of Philosophy in Robotics at The Robotics Institute Carnegie Mellon University, (21 Oct. 1999). 